\title{
Multiple-Scenario Unmanned Aerial System Control: A Systems Engineering Approach and Review of Existing Control Methods
}

\author{
Christopher M. Eaton *, Edwin K. P. Chong and Anthony A. Maciejewski \\ Received: 14 November 2015 ; Accepted: 25 December 2015 ; Published: 4 January 2016 \\ Academic Editor: Javaan Chahl \\ Department of Electrical and Computer Engineering, Colorado State University, Fort Collins, \\ CO 80523-1373, USA; Edwin.Chong@colostate.edu (E.K.P.C.); aam@colostate.edu (A.A.M.) \\ * Correspondence: eatonc@rams.colostate.edu
}

\begin{abstract}
The use of unmanned aerial systems (UASs) in both the public and military environments is predicted to grow significantly. As the demand for UASs grows, the availability of more robust and capable vehicles that can perform multiple mission types will be needed. In the public sector, the demand will grow for UASs to be used for agriculture, forestry, and search and rescue missions. Militaries continue to demand more UAS capabilities for diverse operations around the world. Significant research has been performed and continues to progress in the areas of autonomous UAS control. A majority of the work focuses on subsets of UAS control: path planning, autonomy, small UAS controls, and sensors. Minimal work exists on a system-level problem of multiple-scenario UAS control for integrated systems. This paper provides a high-level modular system architecture definition that is modifiable across platform types and mission requirements. A review of the current research and employment of UAS capabilities is provided to evaluate the state of the capabilities required to enable the proposed architecture.
\end{abstract}

Keywords: Unmanned Aerial System (UAS); autonomous systems; UAS control; path planning; system architecture; Multi-UAS control; collision avoidance; multi-scenario UAS control

\section{Introduction}

In July 2014, the Teal group predicted that worldwide Unmanned Aerial Systems (UASs) expenditures will grow to over $\$ 11$ Billion per year with a total investment of over $\$ 91$ Billion by 2024. It is expected that $86 \%$ of the market will be military and $14 \%$ will be in the civil market $[1,2]$. As the growth continues, challenges and expectations will continue to rise as users will expect more robust and capable vehicles. The military market continues to expand and develop new capabilities and requirements. The growth in the civil market is expected to significantly expand as the rules on civil use of UASs in the US and around the world become better defined. As these markets expand, the need to have systems that can adapt to new missions, sensors, and environments will drive requirements.

The Defense Advanced Research Projects Agency (DARPA) released a Broad Agency Announcement (BAA) for the Collaborative Operations in a Denied Environment (CODE) Program in 2014 [3]. This BAA defines numerous requirements and expectations for future system capability of unmanned and autonomous vehicles working as single systems and multiple vehicle teams. Many of the requirements defined in the CODE BAA can be utilized to define system architecture and capabilities for both military and civilian systems. These requirements will provide a significant portion of the requirements for the system defined herein. In early 2014, DARPA also released a BAA for Distributed Battlespace Management (DBM) that proposes a series of automated and autonomous 
decision aids to assist battle managers and pilots [4]. The DBM envisions, amongst other needs, the ability to enable improved command and control of autonomous operations of UASs, including in manned-unmanned teams. There are multiple thrusts within the DBM program, but one of the primary ones is for improved distributed adaptive planning and control. Additionally, requirements that can be applied globally across UASs from the Federal Aviation Administration (FAA) and other federal, state, and local laws are considered.

There are a significant number of current and future uses for UASs throughout the military and civilian world. The military is currently using, and continues to anticipate increased usage of, these systems in numerous areas including intelligence, surveillance, and reconnaissance (ISR) [5,6], data and communication interfaces [7], electronic warfare [8,9], and limited attack roles [10]. Future cargo and transport capabilities [11] along with search and rescue operations [12] have been envisioned. In the commercial world, there are almost limitless possibilities of uses. Currently, applications exist for agriculture, firefighting, police, sciences, and forestry [2]. Significant efforts in cargo delivery, data capabilities, search and rescue, and traffic information are underway. Evaluating all of these capabilities and needs result in four primary mission types: ISR, persistent loiter, delivery, and attack. These four mission types will define the needs and requirements of the majority of the future UASs across the industry.

An early systems engineering analysis to evaluating the requirements, needs, and capabilities must be performed in an attempt to define a system that can be robust and adaptive to current and future needs. Utilizing requirements defined in the CODE BAA and other resources, a set of requirements can be defined and utilized to develop a system architecture to meet the users' needs. Section 2 provides a high-level problem definition that is addressed by this system architecture design. Section 3 will provide this systems engineering review and architecture definition.

Future multiple-scenario capability will require the system to operate dynamically across one or more of the four mission types and numerous subsets of those missions. Multiple-scenario control algorithms and architectures will enable a single platform to perform multiple mission roles with minimal reconfiguration. A dynamic architecture that enables recognition of sensors, system capabilities, and requirements will ensure the platform enables multiple-scenario support.

Significant work in UAS control and autonomous processes have been performed. The system defined in this paper requires numerous capabilities to be matured, some that already exist and some that need significant development work. Section 4 provides a detailed review of the current state of existing methods and capabilities in UAS autonomous path planning and safety controls. Some of the work that has been completed needs some significant improvement to enable the transition of the capabilities from theory and lab environments to practical applications. Section 5 provides some recommended improvement areas and provides a focus for future planned work by the authors. Final conclusions are provided in Section 6.

\section{Problem Definition}

As the need for future autonomous flight grows and systems mature, a high-level framework of how to design and integrate autonomous systems into existing and new vehicles is needed. Work continues to be performed in developing control capabilities and algorithms required to enable autonomous flight. However, in order for a framework to work, it must provide an architecture that is open and easily modifiable across a diverse type of vehicles and sensor capabilities. The system must enable the autonomous system algorithms to run in a framework that enables maximum flexibility while understanding vehicle capabilities.

\section{System Design}

In order to ensure the ability of autonomous systems to function and be effective across multiple vehicle types, a framework needs to be defined that enables flexibility in design and functionality. A high-level systems engineering review of requirements has been performed based upon the CODE 
BAA [3] and DBM BAA [4] in Section 3.1. A system architecture has been defined that enables the flexibility of design and functionality for autonomous vehicles in Section 3.2. Current and future research needs are briefly discussed in Section 3.3.

\subsection{System Requirements}

The CODE BAA [3] identified four top-level goals for any system proposed and developed:

1. develop and demonstrate the value of collaborative autonomy in a tactical context;

2. rapidly transition the capability to the warfighter;

3. develop an enduring framework to expand the range of missions, platforms, and capabilities that can leverage collaborative autonomy; and

4. develop an open architecture that enables all members of the rich community of unmanned systems and autonomy researchers to contribute to current and future capabilities.

Similarly, the DBM BAA [4] identified a goal of adaptive planning and control that could be distributed across systems to aid a variety of vehicles, weapons, and sensors. The goal is to enable UASs to satisfy the commander's intent while operating in normal or limited communication environments. The ability to have an adaptive decision process across mission types is critical. Hierarchical task processing under limited communication will be an important enabler of autonomy. The autonomous capabilities should be vehicle agnostic. The UASs should be able to negotiate both high-level battle manager tasks and low-level tactical tasks. The capability will need to exist to execute cooperative tasks with other UASs and manned vehicles.

Seven key performance objectives were identified in the CODE BAA where significant improvements are sought and would be critical for any system also developed for the DBM BAA or any other project. Six of the objectives are discussed below. The seventh objective, transition-ability is not considered in this review.

1. Mission Efficiency:

Mission efficiency is an important requirement for both military and civilian operations. The cost of completing the mission needs to be considered. The expense of flying the vehicle along with the duration required to complete the mission are critical concerns for all parties involved. Additionally, the ability to quickly react to changes in mission requirements or system functionality is critical for robust systems of the future. The bulk of the review of current capabilities in existing systems will relate to mission efficiency and control of the system. Mission efficiency can be considered in countless manners, including time to complete the mission (time efficiency), fuel used to complete the mission (fuel efficiency), endurance of mission (endurance), and total number of tasks completed (task efficiency).

2. Communication requirements:

Limited communication frequencies will be available in the future. Limited bandwidth and minimization of communication will be required in future operations and will be a feature for more autonomous vehicles. Additionally, communication in a denied electronic environment will necessitate limited communications. The ability to have communications in unique environments with cognitive capabilities will be required in the future [13].

3. Manning:

Currently, the ratio of operators to vehicles is many-to-one but in the future the desire is to flip the ratio to be one-to-many. To support this change in operational manning, a significant increase in system autonomy must be created. A system must be able to automate its mission path and plan with minimal operator inputs. Hierarchical logic for decision making must be implemented to ensure the most effective completion of the mission and rapid response to mission or system changes. The system must be able to react to both external inputs (operator) and internal inputs 
(system and sensor data). Additionally, unique challenges of training and educating future operators will be critical [14]. The review of current capabilities of algorithms and autonomous features as it relates to mission efficiency will incorporate the considerations of reduction in manning of operations.

4. Command Station:

Future command stations must be robust and provide significant situational awareness for the operator. Being able to command vehicles from a mobile or fixed-based control station will be necessary to ensure flexibility in capabilities. Interfaces that enable the operator to quickly upload new tasks and parameters will be necessary. Limited command station requirements and current capabilities will be addressed in this paper.

5. Openness of the architecture:

Open system architecture is a key of any current and future system viability. To provide a system architecture that can be utilized across multiple system sizes and types it must employ an open architecture to minimize the costs of integration with any existing or new systems. The design proposed in this paper attempts to provide a framework architecture that would satisfy current open architecture standards. Limited review of open architecture requirements will be addressed in this paper.

6. Multi-mission capability:

The ability of a system to perform multiple missions will be critical for future viability. Some airframes may not lend themselves to transition across the four primary mission types of ISR, loiter, delivery, and attack. However, a system that operates primarily in one or two mission areas should be able to perform multiple roles within those mission areas. For example, a system that has a primary role of ISR should be able to perform recurring observation of fixed targets but also be able to transition to tracking of moving targets or persistent observation over a fixed target. The ability of systems to perform multiple missions will be discussed throughout the paper.

\subsection{System Architecture}

In the early 2000s, Boskovich, et al. [15] defined a control architecture for decision making within an autonomous UAS framework. This architecture described a high-level framework for designing autonomous intelligent control systems for UASs. The architecture defined four layers of control: redundancy management, trajectory generation, path planning, and decision making. This general philosophy is still present in many of the design work prevalent today in UAS mission and path planning design. However, this architecture only considers the general control and path planning of a vehicle. To address the high-level requirements previously defined in Section 3.1, a system architecture needs to be developed that can provide the framework for system design and functionality for more than just the mission control of the vehicle. Additionally, a significant portion of the existing research in UAS path planning considers the vehicle a point mass and many of the approaches consider only constant altitude and airspeed. There is minimal consideration of actual vehicle dynamics in the existing research. In order to address the actual vehicle dynamics and utilize those dynamics to improve mission performance utilizing existing methods, an architecture that integrates vehicle dynamics and systems is needed.

Figure 1 provides the high-level architecture defined for our proposed autonomous system. The definition proposes five primary functions: Mission Management, Vehicle Management, Sensors Management, Communications Management, and Safety Management. These five areas provide the sufficient top-level framework for any system, regardless of mission and vehicle type. The advantage of this system is that it provides a modular functionality architecture that can be adjusted for specific vehicles but can be common across numerous vehicle types. The autonomous algorithms will reside within the mission management functionality and will be dependent upon common interfaces and architecture. 
Figure 2 provides a lower-level definition of the system architecture with key critical functionalities within the primary management systems. The functions and capabilities within each management area could be changed depending on each vehicle. However, the interface to the mission management system needs to remain consistent. The key to the architecture is that each primary functional area has a controller that manages the overall function, but capabilities can be added or removed based upon mission and system requirements in a modular fashion without impacting the larger system. Additionally, depending on mission tasking and systems on board, the controller could enable or disable any resident capability to improve performance of mission objectives without changes to the software. The detailed description of each of the five primary functions and their subsidiary functions are provided below.

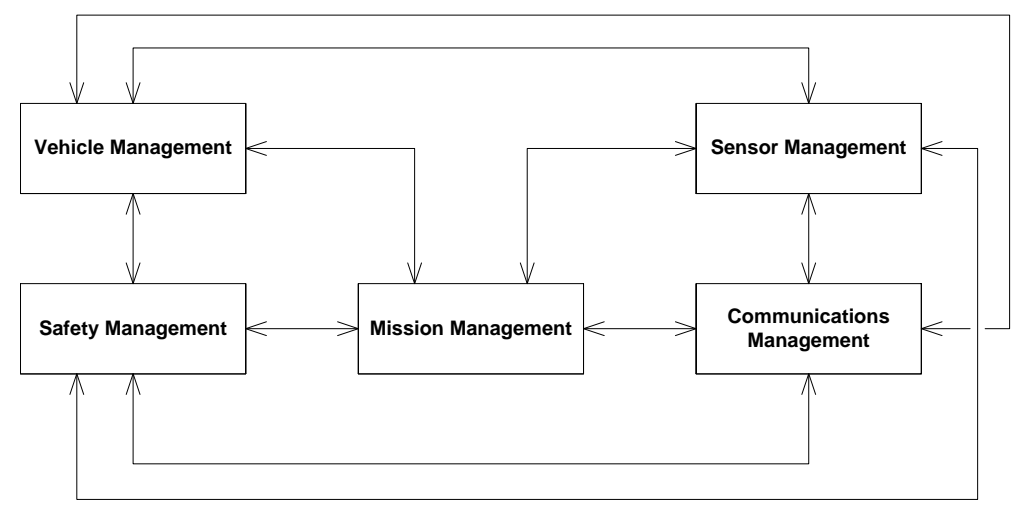

Figure 1. Top-Level System Architecture.

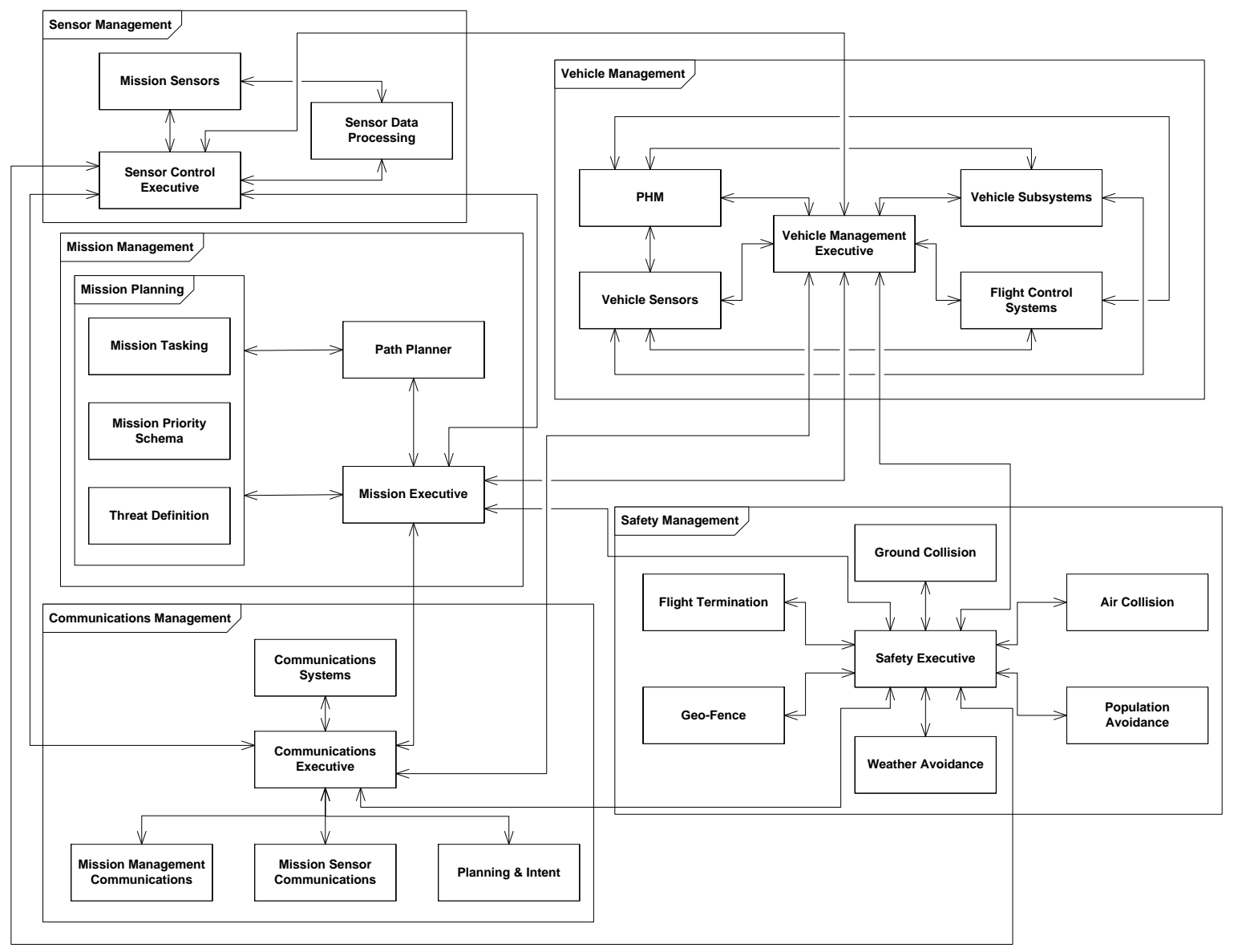

Figure 2. Detailed System Architecture. 
1. Mission Management:

The mission management function is the key to the success of the system architecture. A significant portion of the efforts in this area would be consistent with the work of Boskovic, et al. [15] as discussed earlier. The mission management will provide the primary high-level decision making for the mission performance of the vehicle. The mission management area is the focus of significant research in unmanned and autonomous control. There are three primary functions within the Mission Manager:

(a) Mission Planning:

The mission planning function provides the mission requirement details for the decision-making process of the mission executive. A mission-planning dataset could include definitions of tasks, priorities, and threats. The mission-planning dataset could be uploaded prior to a mission, during a mission, or self created depending on the autonomous capabilities designed within the system. The mission-tasking information would provide the required tasks the system is desired to perform. A mission-priority schema would provide the executive a decision framework to determine which task is of greater priority. For example, tracking a moving target could be defined as a higher priority than general reconnaissance data collection. Threat definition would provide the system considerations for areas to avoid due to known threats as well as considerations for how to handle newly discovered threats. These considerations could include keep-out zones, self protection actions with sensors, or other actions depending upon system capabilities.

(b) Path Planner:

The path planner is the key algorithm for defining where and how the vehicle should move. The path planner utilizes the considerations defined in the mission planning along with information provided (via the mission executive) on system states. The path planning algorithms could be dynamic or changed for any given mission based upon the needs, priority, and other considerations. The path planner must also determine path planning based upon contingency management requirements of the system for subsystem failures. The path-planning algorithm is a significant consideration of this paper and current capabilities are discussed later in this paper.

(c) Mission Executive:

The Mission Executive (ME) is the primary decision maker for the vehicle. The functionality of the ME defines whether to perform the current task defined by the path planner or reacts to safety management information. The $\mathrm{ME}$ also provides and receives communication updates with other vehicles, operators, and other sources as required. The ME commands the vehicle management system to perform flight maneuvers and other vehicle system functionality. The ME could be considered equivalent to a human operator within a manned vehicle system.

2. Sensor Management

Sensor Management provides the control for all the mission sensors installed on the vehicle. Mission sensors are defined as any sensor utilized to perform the mission. Sensors that are used to manage the vehicle control and health are handled within vehicle management. There may be some cross utilization of these sensors for both systems. However, the management of those sensors would be handled by their primary user. Portions of sensor management, such as sensor types and control, are well understood in existing systems. However, the sensor data processing will require continued and significant research to provide autonomous sensor data at a decision level that can be trusted. There are three key functions within the sensor management framework. 
(a) Mission Sensors:

Mission sensors are the sensors specifically installed on the aircraft for data gathering in direct support of mission completion. The mission sensors will be dependent upon the vehicle and mission requirements. These sensors will perform the primary mission duties and could include electro-optical/infrared sensors, radar sensors, radio frequency sensors, or any number of other types. The sensors will have a direct interface to the data-processing module and the control-executive module. These sensors will perform their tasks based upon commands received from the sensor-control executive.

(b) Sensor-Data Processing:

The sensor-data processor will analyze received data and make a decision on the information received based upon algorithms defined. The processing could be utilized for any number of tasks including target recognition, geo-location, target motion, and sensor response. The data will also be processed for transmission, as required, and sent to the sensor-control executive for passage to the communication management for dissemination. A significant level of research is ongoing in areas of sensor data fusion, image processing, and recognition that can support decisions and vehicle tasking.

(c) Sensor Control Executive:

The sensor-control executive (SCE) is the primary controller of all sensors and sensor taskings. The SCE interfaces with the ME and provides sensor availability, sensor capability, sensor data evaluation (target ID, geo-location, etc.), and sensor health. The ME provides the SCE with sensor tasking. The SCE will be required to automatically identify what sensors it has installed on board and what their capabilities are.

3. Safety Management

Safety Management provides overall safety monitoring for the vehicle. The types of safety management performed can be dependent upon vehicle type, sensors installed, capabilities required, and vehicle capabilities. The systems defined in this architecture are notional but are critical for UASs. The safety executive can provide high-priority tasking to the mission executive that can result in overriding current activities for safety reasons. Safety management is an area that is understood, but the integration of it with autonomous systems continues to be researched and developed across vehicle types. The core safety management functions defined in this architecture are explained here, but are not exhaustive of possible functions.

(a) Safety Executive:

The Safety Executive (SE) processes all information from safety management capabilities and provides that information to the mission executive for execution. The SE will prioritize which safety feature should be addressed first (if multiple safety issues are occurring at the same time) and determine the recommended actions.

(b) Collision Avoidance:

Collision Avoidance algorithms for both ground collision and air-to-air would reside within the safety management area. These algorithms would determine when the vehicle is at risk of impacting something and provide recommended action(s) to avoid these problems.

(c) Flight Termination:

Flight Termination is a key issue for unmanned air vehicles. Flight termination can include destructive actions which result in the destruction of the vehicle. However, it can also contain contingency efforts that include immediate landing, reduction in system capabilities, flight-plan alteration, or other functionalities depending upon the mission and range requirements. 
(d) Geo-Fence:

The geo-fence capability defines the areas within or outside of which a vehicle should maintain a presence. There may be unique mission requirements that require a system to fly in certain areas which the geo-fence may not allow based upon changes in mission or knowledge of areas of operation. If the vehicle is approaching a fence limit or has crossed a fence, the safety system should direct the vehicle back within the defined boundary. This geo-fence could be dynamic based upon known aircraft (air collision avoidance), major changes in weather (weather avoidance), or known threats and borders.

(e) Weather Avoidance:

Depending on vehicle capabilities or mission sensor capabilities and requirements there may be a need to avoid undesirable weather. Weather avoidance would provide keep-out areas to the safety executive that could be provided to either the mission executive or the geo-fence capability for management of vehicle path. For sensor functionality issues it would be more critical to provide that information to the mission executive for determination of path route and sensor tasking.

(f) Population Avoidance:

Mission requirements may require the vehicle to perform tasks in areas with significant or critical populations. As a result, there may be a need to fly close to population but avoid interference or impact with people and activities. The population avoidance functionality would determine where the vehicle needs to be to avoid the population of concern and provide the safety executive of how to react to the given situation.

4. Communications Management

Communications Management provides the key interface between the vehicle and other systems. The ability to send and receive both mission information and sensor data can be critical to the success of a given mission. By managing communications separately from the primary processes, it enables changes in communication methods without impacting the underlying functionality of the vehicle. Communications Management will divide the data as either mission management, mission sensor, and planning and intent. There are five primary functions within the Communications Manager:

(a) Communications Executive:

The communications executive provides the primary interface between the mission executive and the installed communication systems. The communication systems installed could vary depending on the vehicle type and mission requirements. The communication executive will provide external communications and data dissemination as required. The system will also need to recognize when communications are not being received for potential operations in a denied environment.

(b) Communications Systems:

The vehicle could have one or multiple communication systems installed for external communications capabilities dependent on mission requirements and vehicle capabilities. Communication types could include line of sight RF, satellite communications, optical/laser or others. Dissemination of data received and to be sent will be via the communications executive.

(c) Mission Management Communications:

Mission management communications will provide mission status, priority, tasking, and threat information for other vehicles. The communications executive will also update this information from any received data for processing via the mission executive.

(d) Mission Sensors Communications:

The mission sensor data will be processed and sent separately from other priority tasks (mission management, planning and intent) to provide external users with specific sensor 
data for analysis and use. By handling the mission sensor data separately from the other data, it prevents critical data being held up by sensor data dissemination. Mission and vehicle tasking data should take priority over data dissemination tasks. This separation will also enable a system to have separate communication systems for data and mission tasks.

(e) Planning and Intent:

The planning and intent data will provide current information on where the vehicle is, where it is going and the intents of its upcoming efforts. This will allow any other vehicles or operators in the mission to monitor and understand the plans of the vehicle. This information will enable users and vehicles to make decisions and recommendations on mission plans and efforts.

5. Vehicle Management

The Vehicle Management system is responsible for the control of the vehicle and systems. The flight control system, vehicle subsystems, vehicle state (via Prognostics and Health Monitoring (PHM) and sensors) all reside within the vehicle management system. Vehicle management is well understood and is standard in most manned and unmanned aircraft. While there is significant research and work ongoing in this area, especially in the areas of PHM and fault tolerant operations, the underlying requirements and architecture are not significantly different from existing platforms. There are five primary areas within the vehicle management system.

(a) Vehicle Management Executive:

The Vehicle Management Executive (VME) manages the vehicle systems control and processing. The mission executive provides the tasking that the vehicle must perform and provide for processing. The data provided is then sent to the flight control systems, vehicle systems, and any other ancillary systems installed that require control. The VME also accepts sensor data and PHM data for processing and determination of whether degraded systems exist and if actions need to be taken. This data is also provided to the mission executive for mission tasking decisions.

(b) Flight Control Systems:

The Flight Control Systems (FCS) of a vehicle can include propulsion, flight control surfaces, flight control sensors, and any other system required for vehicle control. The FCS design and performance is unique to any given vehicle and needs to be provided to the path planner for determination of proper, efficient, and effective path planning.

(c) Vehicle Subsystems:

Vehicle subsystems can include ancillary systems such as electrical, hydraulic, environmental controls, and landing gear. These subsystems provide critical functionality that support the primary flight controls and mission sensors. Subsystems are generally well understood for existing areas but new and improved capabilities (especially in electrical power capabilities) continue to improve the state of these systems.

(d) Prognostics and Health Monitoring:

Prognostics and Health Monitoring (PHM) can provide an estimate of current and future health and capabilities of installed systems. Significant research has been performed and continues to be performed in this area. Fault tolerant design and functions also continue to be researched and can be integrated with PHM functionalities. PHM may or may not be present on a given vehicle but can provide enhanced control and insight into current and future performance.

(e) Vehicle Sensors:

Vehicle Sensors can be numerous and diverse across a vehicle. Depending on the size of the vehicle and criticality of the system there may be minimal or extensive sensing. 
The sensors can include critical flight data such as vehicle speed, rates, and accelerations via air data and/or inertial systems. Sensors can also perform pressure, temperature, voltage, or other critical measurements to support real-time performance or prognostics of future performance. Vehicle sensors continue to evolve and develop based upon new technology and needs.

\subsection{System Needs}

Critical work continues to be performed in all areas of autonomous vehicle systems. The following discussions will provide details on current and ongoing work in selected areas. In the area of vehicle controls there continues to be significant work being performed on flight control systems based upon new and changing vehicle types and control schema. Fault-tolerant systems and PHM continue to be researched and system capabilities need to be improved for future autonomous system use. Sensor capabilities, sensor fusion, and sensor identification capabilities are areas that continue to be researched and will be critical for future use in autonomous systems. Mission management and path planning areas are seeing significant current research and will be required to be developed and become more effective for autonomous system use. Communication system capabilities continue to be researched for improved methods and needs, especially as the available frequency spectrum for communications is reduced for UAS applications. Safety management will continue to see research and growth as autonomy grows in order to improve and ensure trust of these autonomous systems.

\section{Review of Existing Methods and Capabilities}

There are significant efforts ongoing in all areas of UAS autonomous control. This section will provide a review of the current state of path-planning and critical safety control as they relate to UAS autonomous controls. Section 4.1 provides a review of the current state of path planning algorithms that directly support the mission management capabilities defined in Section 3.2. Section 4.2 examines the state of critical safety control features that ensure safe flight, which directly support the safety management capabilities defined in Section 3.2.

\subsection{Path Planning}

Path planning requires knowledge of target or mission needs in order to properly complete the planning algorithms. We will define three primary types of path planning: Fixed Target, Moving Target, and Target Search and Surveillance. Additionally, a system that can complete multiple scenarios within the three primary areas is valuable. An extension to multiple scenarios would be multiple aircraft supporting either the same type of mission or multiple scenarios.

\subsubsection{Fixed Target}

Fixed target path planning deals with the algorithms utilized with visiting fixed locations for information gathering or support. UASs are currently being used in missions that require the vehicle to visit a set of targets and maintain an optimum flight path to complete their tasks.

Many of the fixed target path planning problems can be considered similar to the traveling salesman problem (TSP) that has been evaluated significantly in numerous ways for decades since Dantzig et al., developed a solution as an integer linear program [16]. The TSP type problem has been approached with multiple solutions. Many of the evolutionary algorithms used to solve these NP-hard (short for non-deterministic polynomial-time hard, widely taken to imply that the problem is computationally intractable [17]) problems provide acceptable results, although many of them have constrained the problems to some level.

Early approaches in solving the path planning problem included the use of a Tabu Search (TS) heuristic algorithm $[18,19]$. The TS can provide a solution that allows for progression without becoming trapped in local optima. Ryan, et al. [20] used a Reactive Tabu Search method to solve UAS routing in the construct of a multiple Traveling Salesman Problem with time windows. Their objective 
was to maximize expected target coverage while incorporating weather and a survival probability at each target as random inputs. Wang et al. [21] proposed a Tabu (Taboo in their paper) Search algorithm for multiple task planning for multiple UASs that showed better performance than genetic algorithms or ant colony optimizations. Zhao and Zhao [22] utilized a Tabu search algorithm to develop their path and time. Numerous methods have been developed more recently that provide better results to the path-planning problem than the Tabu Search, although it still is valuable for certain processes. Additionally, TS algorithms are only useful for small problems that do not consider vehicle availability or tasks that require constraints beyond simple time ordering.

In 1956, Edsger Dijkstra proposed an algorithm for finding the shortest path between two points [23]. This algorithm is a key method for finding shortest paths for robots and unmanned vehicles and is used for numerous applications. The use of Dijkstra's algorithm can be found in countless applications to UAS path-planning applications [24-29].

Tong et al. [30] proposed a method of path planning that utilized Voronoi Diagrams and Discrete Particle Swarm Optimization (DPSO). A Voronoi diagram depicts lines that are equidistant to the closest neighboring points of interest, resulting in areas that define all points closest to the points of interest. A Voronoi diagram works similarly to and in conjunction with Dijkstra's algorithm. The lines from the Voronoi were used as an initial path, with the points of interest being threats to be avoided. A DPSO algorithm was then used for simultaneous target attacks by multiple vehicles.

Receding horizon control (RHC) [31] is a feedback control technique, also referred to as model predictive control, which is used across a large variety of applications. Receding horizon control is used as part of numerous algorithm types for UAS path planning. The advantage of RHCs is that it enables control of systems with a large number of inputs and outputs, especially for systems with complex objectives and strong nonlinear dynamics and constraints. The use of future considerations and predictions while optimizing the current time requirements is the key feature of RHC. Multiple path-planning algorithms utilize a form of RHC as part of their planning processes. The RHC control scheme has become useful for UAS algorithms due to the limited requirements for computational resources when compared to algorithms that perform global planning methods. Kuwata et al., developed a decentralized RHC for multi-vehicle guidance [32,33]. Xiao et al. [34] used an RHC method in conjunction with a virtual force method to improve the performance of the RHC. Peng et al. [35] developed a cooperative search algorithm utilizing RHC with a rapidly exploring random-tree path-planning algorithm. Schouwenaars et al. proposed a multiple aircraft trajectory planning algorithm utilizing a RHC strategy with a mixed integer linear programming basis [36]. There have been multiple efforts utilizing RHC methods in conjunction with Partially Observable Markov Decision Processes [27,37-39].

In 1995, Kennedy and Eberhart [40] proposed a methodology of nonlinear function optimization using particle swarm optimization (PSO). This method provides a simple and computationally useful algorithm for optimizing a wide range of functions. The use of PSOs as part of a UAS path-planning algorithm has been employed successfully by numerous researchers [30,35,41-44]. Roberge et al. [45] provided a comparison of GAs and PSOs for UAS path planning. The resultant of the comparison shows that the GA produces superior trajectories to the PSO.

An approach that has shown good results is the use of Genetic Algorithms (GA). A GA provides a heuristic method based on natural evolution by defining the decision variable as a chromosome. The chromosomes defined by the problem give the resultant population and an algorithm is utilized that generates an evolution process until a satisfactory solution results. Sahingoz $[46,47]$ and colleagues $[48,49]$ have performed significant work in using GAs for both single and multiple UAS path planning which has shown satisfactory results. Cheng et al. [50] developed an immune genetic algorithm that provided an "immune operator and concentration mechanism" that improved convergence of existing GA algorithms. GAs can, under certain circumstances, suffer from premature convergence. Price and Lamont [51] used a GA design for self-organized search and attack of UAS swarms. Pehlivanoglu [52] proposed a vibrational GA algorithm enhanced with a Voronoi Diagram 
in an effort to improve the convergence problem. Research is continuing in the use of GAs for path planning of UASs $[53,54]$.

An algorithm based upon the annealing of metal [55,56] can be utilized to find global minimum of an objective. Drawing upon the annealing process, a Simulated Annealing (SA) algorithm will search randomly in the area of an initial guess. If an improvement is found, the new value is kept. If deterioration is noted, the result may be discarded or kept depending upon a temperature-dependent probability. A cooling schedule is used to determine when the temperature has been sufficiently cooled from the initial value. Turker et al. [57] presented a method for 2D path planning in a radar threat constrained environment using a simulated annealing algorithm. Leary et al. [26] evaluated five algorithms including SA, Consensus Based Bundle Algorithm (CBBA), greedy allocation, optimal Mixed Integer Linear Programming (MILP), and suboptimal MILP. The results showed that the SA algorithm provided the best solutions for path generation but required the longest computation time of the five algorithms; however, the growth in computation time with increased parameters was the lowest.

In 1992, Marco Doringo proposed an approach for finding an optimal path that drew upon the behavior of a colony of ants [58]. The ant colony optimization (ACO) approach has been adopted as a method to optimize UAS path planning. Fallahi et al. [59] proposed a method that integrated $\mathrm{ACO}$ and an analytic hierarchy process that showed good results for path planning using the ACO algorithm. An adaptive ant colony optimization approach for multiple UASs for coordinated trajectory re-planning was proposed by Duan et al. [60]. An extension of ACO looks more generically at digital pheromone responses and has been used to improve target search methods [61-63]. Shang et al. [64] proposed a hybrid algorithm that utilized GA and ACO algorithms for Multi-UAS mission planning which provided performance improvement over the two independent methods.

\subsubsection{Moving Target}

Moving Target path planning deals with the algorithms utilized to find and follow moving targets. Less work has been performed in the area of moving target tracking as compared to fixed target tracking. However, several similar algorithms to fixed target tracking, including Partially Observable Markov Decision Processes (POMDPs) and Genetic Algorithms (GAs), have been used with some success for finding and tracking moving targets.

Krishnamoorthy et al. $[65,66]$ developed a method of searching and tracking a moving target traveling with a known speed and direction on a road network while utilizing unattended ground sensors for target detection. This work was later developed and demonstrated with multiple-vehicles, multiple-targets, and a large series of ground sensors by Rasmussen and Kingston [67]. This approach relies upon unattended ground sensors to trigger when a moving target passes it's location. The sensor then informs the UAS of an intrusion and the associated information required to search and track the intruder. The system has shown some limitations due to sensor false alarms and delay in sending information due to limited line of sight data transmission capability. However, the functionality shows promise in supporting a network of ground sensors and vehicles to monitor roads or perimeters for intrusion.

Moon et al. [68] proposed the use of probability density functions in coordination with a negotiation task assignment framework for UAS tasking. The algorithm uses information gathering-based task assignment with a two-layer framework. An information-gathering layer uses the probability density functions to generate minimized value future trajectories. The task assignment layer utilizes a negotiation-based task allocation to assign tasks to the UASs in the network. Results showed promising results to search an area with minimal overlapping while finding all targets being searched.

Xiao et al. [34] proposed a virtual force and receding horizon method that enabled multiple-UAS cooperative search in a fixed region for unknown moving targets. Virtual force algorithm alone can be limited by being trapped at local minima while the receding horizon has large computational requirements that limits the length it can look ahead. The algorithm presented combined the two methods in order to alleviate the limitations of each method. 
Sun and Liu [69] proposed a modified diffusion-based algorithm to manage target uncertainty while controlling multiple UASs with a hybrid receding horizon/potential method algorithm for a coordinated search for a moving target. The search area was divided into cells and the algorithm coordinated vehicle search tasks based upon weighting of cells of the search region. The cells not searched that were closer to a given UAS were given a higher weighting than ones closer to a different UAS. A hybrid method that combined potential and receding horizon methods was used to reduce the computational burden.

Frew et al. [70] and Summers et al. [71,72] proposed similar control algorithms for multiple UAS coordinated standoff tracking of moving targets by utilizing Lyapunov guidance vector fields. Both approaches utilized Lyapunov guidance vector fields to generate stable paths for the UASs to fly while tracking a moving target. Multiple-UASs could be used by phasing them around the vector field solution. Both approaches showed acceptable results for multiple vehicles orbiting and tracking a moving target.

Geyer [73] proposed a method for urban searching of a moving target that considered complex geometry from buildings that can impact the ability of the sensor to see the target. The method utilizes search trees and particle filters to evaluate path options and provides efficient filtering along with a method of compressing the visibility function.

Bertuccelli and How [74] propose a Markov chain-like model for target motion estimation approach similar to particle filtering in order to account for the uncertainties in the target location estimates. Stochastic simulations of realizations of the transition matrix with posterior distribution approximation enable easy re-sampling of the posterior distribution. This method is valuable for searching for moving targets where the models of the target motion are poorly known.

Ragi and Chong [39,75] proposed a method of UAS control utilizing POMDPs for tracking moving targets including evasive targets and threat avoidance. The resulting algorithm enabled a vehicle, or multiple vehicles, to be able to track moving targets. The design was robust enough to be able to track an evasive ground vehicle as well as avoid threats, obstacles, and other friendly vehicles while maintaining tracking of the target. Wind compensation and variable speed and altitude capabilities were integrated as well.

\subsubsection{Target Search and Surveillance}

Target search path planning deals with searching for targets with no or minimal information on the target of concern. Surveillance deals with repeated coverage and search of a specified area to obtain the desired information. Search problems are generally defined by generating a grid of cells over an environment. Poor information about target locations and noisy sensors can increase the difficulty of quickly and easily finding targets.

One regional surveillance method to ensure maximum coverage is the lawnmower path definition, sometimes referred to as a boustrophedon pattern. The pattern is efficient for ensuring maximum coverage of an area. However, it is very time consuming, and depending on the requirement of the mission, may be ineffective for the needs of the operator. Similarly, a spiral pattern that slowly spirals in either smaller or larger radius could provide similar results.

One challenge is determining how long or how many times a vehicle must survey a point before a satisfactory level of confidence that a target exists in a given area. Bertuccelli and How [76] proposed a robust UAS search method for determining target existence with the consideration that the prior probabilities for a given cell are poorly known. The use of Beta distribution enabled a prediction of the number of searches required in a given cell to achieve the desired confidence that a target exists in a given area.

Qu et al. [61] proposed a pheromone-based algorithm with an artificial potential field to perform regional surveillance with multiple UASs. A region would be separated into multiple units and a pheromone model would be applied to each unit. Pheromones have a diffusion feature that results in a portion of its information being translated to the units around it, using either an attractive or 
repulsive factor. This information then results in a gradient of pheromones being formed providing a path for the UAS to follow. An artificial potential field was then used to aid in obstacle avoidance, collision avoidance, and optimal search.

A planning algorithm by Song et al. [77] for optimal monitoring of spatial environmental phenomena based on Gaussian process priors showed improvement at finding global maximum conditions. This algorithm would be valuable for surveying unknown spatio-temporal fields such as gas plumes and humidity. Lee and Morrison [78] propose a search algorithm for multiple-vehicle maritime search and rescue that accounts for target drift using a mixed integer linear program, relying on a model over multiple periods to account for object location over time.

Zhang and Pei [79] developed a method to track the boundary of an oil spill using model predictive control and universal kriging. Universal kriging is an interpolation technique closely related to regression analysis. By combining universal kriging and model predictive control they proposed a method to search the environment with a sensor and, based upon the initial samplings, develop a means to track the boundary of the oil spill.

Hu et al. [80] provided a multi-agent information fusion and control scheme for target searching. An individual probability map for target location(s) was maintained by each vehicle and updated, based on measurements made by the vehicle, using Bayes' rule. A consensus-like distribution fusion scheme, updated with asynchronous information, was used to create a multi-agent probability map for target existence. A distributed multi-agent coverage control method for path planning, using a Voroni partition, that ensured a sufficient number of visits to each cell was performed.

Hirsch and Schroeder [81,82] proposed a method of decentralized cooperative control of multiple-UASs performing multiple tasks in an urban environment. The construct assumed limited communication between the vehicles and considered potential line of sight impacts from buildings. The method required each UAS to perform independent receding horizon feedback control that relied on its own information along with any received remote information from neighbor vehicles to plan the required search path.

\subsubsection{Multiple Objective}

As a vehicles mission progresses, the need to adapt to new information or to change objectives may be required. Additionally, balancing multiple internal objectives such as path length, endurance, and safety present challenges to the algorithm development and usage for UAS control. The ability of a system to perform mission re-tasking and path re-planning is needed to enable multiple-objective scenario use. Numerous path planning algorithms discussed earlier integrate object avoidance algorithms. Other safety considerations (such as air collision avoidance) would require unique algorithms that are discussed separately.

Multiple-objective path planning deals with integrating multiple path-planning algorithms into one framework to enable a vehicle to perform multiple missions, either in a hierarchical fashion or simultaneously. Multiple-scenario response can be due to the need to respond to changing environments, as seen in Meng et al. [24]. They proposed a hierarchical approach that removed and replaced mission objectives as the mission requirements are changed or canceled. The approach developed an initial path generation for each vehicle and then, as requirements changed, re-allocation of objectives was performed with each UAS receiving a unique tasking and path generation.

Hirsch and Schroeder [81,82] defined a solution for vehicles performing tasks of searching for targets while also tracking targets already found with a hybrid heuristic algorithm that combined a greedy randomized adaptive search procedure with simulated annealing (GRASP-SA). The UASs were provided no knowledge of where or how many targets were present in the environment. At each decision point, the UAS was required to determine whether to continue searching for new targets or track the targets already detected. The approach was performed in an urban environment model, incorporating object avoidance and line-of-sight obstructions into the decision 
process. The GRASP-SA algorithm was successfully applied to the problem set and provides a unique approach to the multiple-objective problem for search and tracking of multiple targets.

The use of Tabu search as a method for multiple-objective planning was proposed by Wang [21]. This early evaluation of the problem requires additional work but showed promise as a way to plan missions for multiple-task planning with goals of maximizing number of completed tasks with a minimization of range and time. The Tabu search algorithm is used to optimize the task allocation scheme after a planning model is built. The problem set evaluated was simple, and requires a more complex and practical environment to be evaluated in order to determine the overall value of this approach.

An algorithm utilizing multi-criteria decision making cost functions and multi-attribute utility theory to make complex decisions for vehicle path planning was designed by Wu et al. [83] with a focus on UAS delivery of medical supplies while flying in a complex airspace. The approach focused on flying a vehicle under existing visual flight rules in the national airspace, which continues to be a critical concern. For en-route planning, multiple criteria were considered within the cost construct of the algorithm: time, fuel, airspace classes, aircraft separation risk, storm cell risk, cruising levels, and population risk. These concerns, while more tactically focused on completing a singular mission type (medical delivery), could be transformed into other objectives in a similar construct for more complex efforts.

Ilaya [84] proposed the use of a decentralized control scheme involving multiple vehicles performing a multi-objective trajectory tracking and consensus problem using particle swarm optimization. The approach incorporated a two-level decision process: a high-level supervisory level and a local vehicle control level. Decentralized model predictive control was utilized for the vehicle-level synthesis of cooperative and self behaviors. A Lie group of flocks approach was used for the high-level supervisory control decision making. In related work, Ilaya et al. [85] provided an approach for distributed and cooperative decision making for collaborative electronic warfare. Similar algorithms were utilized with a focus on radar deception, ensemble tracking, and collision avoidance among the vehicles.

Optimizing resources for multi-criteria decision making using ant colony optimization (ACO) and analytic hierarchy process (AHP) was proposed by Fallahi et al. [59]. Unlike other approaches that rank a finite set of alternatives in a multi-criteria decision making problem, this approach utilizes the ACO to obtain optimal solutions satisfying some of the path-planning criteria. The AHP is then used to select the best UASs to perform each portion of the mission, optimizing the results of the overall mission. This approach could be extended to numerous UAS problems and objective constructs.

Peng et al. [86] proposed using a linkage and prediction dynamic multi-objective evolutionary algorithm in conjunction with a Bayesian network and fuzzy logic decision making process. Historical Pareto sets are collected and analyzed for the online path planning. A Bayesian network and fuzzy logic are then utilized for bias calculations for each objective. Results of using this method shows improved performance over completely restarting the path-planning algorithm at each objective change.

\subsubsection{Multiple Aircraft}

Multiple aircraft path planning deals with both centralized and decentralized coordinated mission efforts of multiple vehicles. Significant research is currently being performed in this area and could constitute a full survey paper on its own. The goal of this section is to highlight some of the primary methods being proposed for single and multiple task allocation that show significant capabilities of interest. Many of the previously discussed methods incorporated multiple vehicle controls into their algorithms, and are not repeated here. Specifically, the algorithms discussed in Section 4.1.4 also supported multiple-aircraft control.

Swarming is not considered in detail for this review as the controls for swarming have been extensively reviewed $[87,88]$ and are generally focused on multiple vehicles working towards a single task while acting in a more biological-system manner. The term swarm is used in multiple ways 
currently to describe different operations. For this paper, a swarm is a group of vehicles working towards a common task in a group manner. This section focuses on multiple-UASs performing unique cooperative single and multiple-tasks in a controlled but decentralized environment.

One significant area of research has been at MIT under Professor Jonathan How. An unbiased Kalman consensus algorithm was proposed by Alighanbari and How [89]. Consensus-based algorithms proposed by How and associates consider both a decentralized consensus-based auction algorithm (CBAA) and the consensus-based bundle algorithm (CBBA). The CBAA is used for single-assignment tasking of single agents using an auction with greedy heuristics and a conflict-resolution protocol for consensus on winning bids for allocation [90]. The CBBA algorithm allows each agent to bundle assignments awarded as with CBAA but enables the system to collect and perform multiple assignments [91,92]. Both algorithms show significant value in allocation of tasks and the overall performance of the system of UASs to complete missions in complex environments.

A survey of early consensus problems for multi-agent coordination and development of consensus seeking algorithms was completed by Ren and Beard [93-95]. Extensions of some of this work have included forest fire monitoring using multiple UASs [96] and perimeter surveillance using teams of UASs [97].

Zhao and Zhao [22] propose task clustering as a means to divide a large portion of tasks among multiple UASs. Ou et al. [98] propose a chaos optimization algorithm for task assignment to multiple-UASs. Zhang et al. [99] propose a cooperative and geometric learning path-planning algorithm for single and multiple UASs that attempts to minimize both the risk and length of the path flown by the vehicle(s).

\subsection{Safety Controls}

Safe control of UASs is a critical area of concern. Manned aircraft have the unique advantage of having a pilot in the loop directly at the vehicle with the ability to react to safety concerns immediately. UASs require either automated response capabilities or the ability to quickly provide critical information to an operator for response. The notion of run-time assurance to provide confidence in autonomous decisions is a critical area of concern. Collision control is a huge concern for UASs, which has resulted in work on air, ground, and object avoidance. Boundary and population control work has focused on keeping UASs in controlled areas and out of the area of risk to populations. Weather avoidance enables a vehicle to evaluate the weather and determine whether the flight path should be modified autonomously. Fault tolerance, isolation, and prognostics is a large research area focused on enabling vehicles to continue to operate under less than ideal functionality. Flight termination is a major concern for how to manage vehicles that are not operating properly and must immediately cease flight operations. Test safety is a unique area of concern focused on how to properly test vehicles prior to release to normal operations where the likelihood of failure is higher and risks can be increased.

\subsubsection{Run-Time Assurance}

A critical safety concern for autonomous systems is trust in decision making. As the ability of autonomy to make decisions, the state space of the system grows so large that it is impossible to verify and validate all possible decisions made by an autonomous system. As a result, a way to ensure that the system does not make decisions outside an acceptable region is required. The concept of run-time assurance has been investigated by the Air Force Research Lab [100,101] and is supported by research by Barron Associates [102]. Mark Skoog at NASA Armstrong has proposed an Expandable Variable-Autonomy Architecture (EVAA) system architecture that would enable confidence in UAS decision making by bounding the decision making to prevent a system from operating in unsafe manners [103]. Research continues to be performed in trust of autonomous systems, including in manned systems, such as autonomous ground collision avoidance systems on fighter aircraft [104-107]. Extending the confidence in autonomous unmanned systems will be critical in the future. 
Ensuring that the system architecture enables safety, including implementing a run-time assurance concept as in the EVAA architecture, will be critical to ensure safe operations.

\subsubsection{Collision Avoidance}

Collision control has been a key concern for all aircraft, but is more difficult on a UAS due to the lack of onboard pilots. The vehicle must be able to avoid collision with the ground, with other vehicles, and any objects it may encounter. Developing algorithms to perform vehicle maneuvering for collision avoidance can be performed by methods previously discussed in Section 4.1. The difficulty in collision avoidance is identification of the risk and determining what mitigation must be performed. The challenge is identifying what sensor or model is required to determine the risk and what mitigations can be implemented.

Ground collision avoidance and recovery systems were originally developed for manned aircraft. In 1990 a patent was granted for an "Aircraft ground collision avoidance and autorecovery systems device" [108] which provided a system design for calculating aircraft flyups to prevent ground collision. Automatic Ground Collision research efforts [109-112] continued in the USAF and NASA for years. In early 2015, an Automatic Ground Collision Avoidance System installed on the F-16, which was based upon the earlier research by the USAF and NASA, saved the pilot and aircraft during operations against the Islamic State in Syria [113]. Recently, NASA [114] integrated a similar system on a small UAS with a smart-phone interface for ground collision avoidance capabilities. These systems rely upon an onboard digital terrain elevation model of the earth to calculate threat and recovery, nullifying the need for additional sensors on the vehicle. Avoiding colliding with stationary objects is an additional consideration in the ground collision avoidance area. Scherer et al. [115] proposed implementation of Laplace's equation to develop a potential field solution. Kobilarov [116] utilized a cross-entropy method in conjunction with rapidly expanding random trees for object avoidance path generation. Hrabar [117] proposed using an expanding elliptical search method to determine paths for object avoidance.

Air collision avoidance of both aircraft and stationary objects is a more difficult problem to solve. A patent was filed in 2001 [118] that provided the initial concept of a safety zone sphere around a vehicle that required both passive and active sensors to monitor the safety zone. Once incursion was detected, the system would inform an onboard sense-and-avoid computer for corrective action response. This underlying philosophy has been utilized in numerous research efforts. One of the critical issues for air collision avoidance is awareness of air traffic within the local airspace. For vehicles that do not have sufficient sensors or capabilities, a ground-based sense-and-avoid system (GBSAA) has been proposed for numerous aircraft types [119]. The Department of Defense, under an Army program, is in the process of developing and deploying several GBSAA systems in specific areas around the US [120]. However, there are challenges to using GBSAA for UAS operations, including the potential that they may not be able to actually support collision avoidance between vehicles [121]. The additional disadvantage of a GBSAA system is that it still requires integration with the UAS or have a pilot in control of the vehicle to make avoidance decisions. Maneuver algorithms are rather straightforward to implement depending on the path requirements; however, integration of GBSAA sensor data is more difficult. Most sensors installed on vehicles or the ground are only sensing other vehicles that may threaten the vehicle of concern. The integration of that information along with vehicle path planning and control for collision avoidance is required. One approach for being able to identify where all traffic is for collision avoidance sensing is the requirement by the FAA to have Automatic Dependent Surveillance-Broadcast (ADS-B) on all aircraft by the year 2020 [122]. Lin and Saripalli [123] proposed a method of collision avoidance utilizing ADS-B with a greedy rapidly exploring random tree.

Numerous methods have been proposed and explored as ways to prevent collisions between UASs and other aircraft. Considering collision avoidance an optimization problem and implementing geometry-based solutions has been proposed by multiple sources [124-126]. Lin and Saripalli [127] 
proposed using reachable sets, a collection of locations that can be reached at a given instant in time, for collision avoidance. Lin and Saripalli [128] also proposed using a variation of a rapidly expanding random trees approach with 3D Dubins Curves to avoid both stationary and moving targets. Optimal control methods were proposed by Shim and Sastry [129] and Bareiss and van den Berg [130] that require accurate system models to be implemented. Methods utilizing Markov decision processes were proposed in [131-133] but can be limiting due to the large state space requirements. Jackson et al. [134] propose a sensor suite of both onboard and offboard sensors with sensor fusion as a method to detect threats. Numerous methods of integrated sensor solutions exist for identifying air collision threats including Traffic-alert and Collision Avoidance System (TCAS) [135], mobile radar [136], electro-optical/infrared [137], and Laser \& Light Detection and Ranging (LIDAR) [138]. Angelov [139] provides a significant review of the work being performed in this area. In 2009, the US Office of Naval Research performed a detailed study of sensor solutions for sense and avoid [140].

\subsubsection{Boundary Control}

Geofence is a general concept of providing boundary control of where a vehicle can operate. For UASs this can include ceilings and floors as well as walls of operation. Geofencing is seen as one means of aiding in the sense and avoid issue of UASs, especially small UASs. Stevens et al. [141] proposed a geofence system that was platform independent. Many of the small UAS flight controls systems, such as Arduino [142], include a geofence capability inherent in its system that can be utilized for safe operations. Hayhurst et al. [143] propose that standards be developed for assured containment that provides an independent capability separate from the geofence algorithms that are generally resident internal to system software.

\subsubsection{Test Safety}

The Range Safety Group of the Range Commanders Council has provided guidelines for Flight Safety Systems for UAS Operations within defined range locations [144]. This standard provides guidelines for safe recovery of UASs that are recommendations for flying on these ranges. Johns Hopkins Advanced Physics Laboratory has proposed a framework for safe testing, Safe Testing of Autonomy in Complex, Interactive Environments (TACE), that can provide both safety features and complex interactive environments in a virtual fashion enabling improved safety and testability [145]. Emergency recovery and flight termination requirements are a necessary capability of UASs, especially larger class UASs that can cause danger to property or personnel. A technology survey of these systems by Stansbury et al. [146] provide a good overview of the recovery and termination systems. The need to terminate or provide emergency recovery is critical, especially when testing unproven systems. Integrating emergency recovery and/or flight termination capabilities with systems such as TACE for testing of vehicles will be a critical enabler to safety of the system and any property and personnel close to the test area. Including enabling features like geofencing/boundary control, collision avoidance techniques, reversionary modes to disable autonomy algorithms, and the ability to take over the vehicle during undesirable operations will be critical for safe testing of these systems. The size and level of implementation of recovery and termination systems along with systems like TACE and EVAA along with considerations of run time assurance will be dependent upon vehicle size and risk.

\section{Improvement Areas}

The use of UASs will continue to grow in the future. To be able to address growing and changing needs in the future, a system will need to be able to adapt and change to new and emerging requirements. A significant amount of work has been performed to develop algorithms that will enable path planning for multiple mission types. As discussed, the majority of this work has been performed with key assumptions about vehicle capability and simplified performance metrics. In order to improve the capabilities of these systems, the integration of system capabilities and 
performance must be included in the algorithms. Development of controls for UASs that include estimates of vehicle performance and capabilities will be key to improving the usefulness of the algorithms in actual systems. To date, limited evaluation of existing algorithms with multiple vehicle types and their associated dynamics has been performed. Additionally, integrating vehicle performance capabilities and limitations into the algorithms will result in a better understanding of the capabilities and value of the different algorithms in realistic implementations.

The ability to integrate multiple mission types in a single vehicle will be critical as development costs and time lines continue to challenge the market. Developing system designs and algorithms to account for multiple-scenario missions will enable the growth of UASs with reduced delay and costs. Development of a hierarchical scheme for both single and multiple UASs needs to continue in order to enable future systems. The integration of key system safety requirements, especially in the area of collision avoidance and boundary control, will help ensure that these vehicles are safe and trustworthy to operate. Developing overarching safety architectures that ensure safe operations will be critical to building trust in autonomy and enabling run-time assurance. Ground collision avoidance is becoming standard with new capabilities in operation, but air collision and object avoidance still has development work to be performed to ensure safe and trusted operations.

While not discussed in detail in this paper, sensors and sensor data fusion will be critical areas that need to be addressed to truly enable autonomous control methods. Until the UAS can self evaluate the data that it has received and make the appropriate decisions on that data, the usefulness of the vehicles will be mostly limited to man-in-the-loop operations. The size and capability of sensors, and the vehicles they will be integrated in, will be critical to the growth and development of UASs for future use.

\section{Conclusions}

The development of autonomous UASs and subsystems will continue to expand in the future. The systems being developed will need to be safe, reliable, and effective across multiple operational environments and tasks. Development of systems that can perform multiple scenarios and be adaptable to new capabilities and responsive to changes in environments and missions will be key to future success. Significant research and development in capabilities has been performed and continues. A modular system architecture was proposed in this paper that will enable safe and trustworthy performance of multiple-scenario missions. Critical path-planning and safety controls which will provide underlying system capabilities for future development were reviewed. Future integration of actual UAS performance capabilities, including costs for vehicle dynamics, into the proposed architecture and evaluation of numerous path-planning algorithms will enable a better understanding of existing methods and future capabilities on actual systems.

Author Contributions: The primary contribution for the article and analysis was performed by Christopher M. Eaton. Support for the work, analysis, and written document was overseen by Edwin K. P. Chong and Anthony A. Maciejewski. All authors had contributed to setting the work and analysis in this paper, as well as proof reading the final document for publication.

Conflicts of Interest: The authors declare no conflict of interest.

\section{References}

1. Teal Group Predicts Worldwide UAV Market Will Total \$91 Billion in Its 2014 UAV Market Profile and Forecast. Available online: http://www.tealgroup.com/index.php/about-teal-group-corporation/press-releases / 118-2014-uav-press-release (accessed on 19 August 2015).

2. Valvanis, K.; Vachtsevanos, G. Future of Unmanned Aviation. In Handbook of Unmanned Aerial Vehicles; Springer: Dordrecht, The Netherlands, 2015; pp. 2993-3009.

3. DARPA Collaborative Operations in a Denied Environment (CODE). DARPA-BAA-14-33. Available online: https://www.fbo.gov/spg/ODA/DARPA/CMO/DARPA-SN-14-28/listing.html (accessed on 30 November 2015).

4. DARPA Distributed Battle Management Program. DARPA-BAA-14-17. Available online: https://www.fbo.gov/spg/ODA/DARPA/CMO/DARPA-BAA-14-17/listing.html (accessed on 30 November 2015). 
5. Otto, R.P. Air Force ISR 2023: Delivering Decision Advantage; Headquarters United States Air Force: Washington, DC, USA, 2013.

6. Dempsey, M.E. Intelligence, Surveillance, and Reconnaissance Joint Force 2020 White Paper; U.S. Army: Washington, DC, USA, 2014.

7. Seffers, G. Joint Aerial Layer Network Vision Moves Toward Reality. Available online: http:/ / www.afcea.org/content/?q=node/11123 (accessed on 19 August 2015).

8. Schechter, E. UAVs Could be Next Step for Electronic Warfare. Available online: http:/ / archive.c4isrnet.com/article/20140507/C4ISRNET08/305070006/UAVs-could-next-step- electronic-warfare (accessed on 19 August 2015).

9. Cevik, P.; Kocaman, I.; Akgul, A.; Akca, B. The Small and Silent Force Multiplier: A Swarm UAV-Electronic Attack. J. Intell. Robot. Syst. 2013, 70, 595-608.

10. Callam, A. Drone Wars: Armed Unmanned Aerial Vehicles. Int. Aff. Rev. 2010, 18, 3.

11. Lockheed/Piasecki Team Tackles Cargo UAV. $2014 . \quad$ Available online: http:/ /aviationweek.com/awin/lockheedpiasecki-team-tackles-cargo-uav (20 August 2015).

12. Myers, M. New funds to aid coast guard in adopting a UAV. Navy Times, 25 April 2015.

13. Reyes, H.; Gellerman, N.; Kaabouch, N. A Cognitive Radio System for Improving the Reliability and Security of UAS/UAV Networks. In Proceedings of the 2015 IEEE Aerospace Conference, Big Sky, MT, USA, 7-14 March 2015; pp. 1-9.

14. Ogan, R. Educating the Next Generation Engineers for Unmanned Aircraft Systems Applications and Challenges. In Proceedings of the SoutheastCon 2015, Ft. Lauderdale, FL, USA, 9-12 April 2015.

15. Boskovic, J.D.; Prasanth, R.; Mehra, R.K. A Multilayer Control Architecture for Unmanned Aerial Vehicles. In Proceedings of the American Control Conference, Anchorage, AK, USA, 8-10 May 2002; pp. 1825-1830.

16. Dantzig, G.; Fulkerson, R.; Johnson, S. Solution of a Large-Scale Traveling-Salesman Problem. J. Oper. Res. Soc. Am. 1954, 2, 393-410.

17. Garey, M.R.; Johnson, D.S. Computers and Intractability: A Guide to the Theory of NP-Completeness; Freeman: New York, NY, USA, 1985.

18. Glover, F. Tabu Search: A Tutorial. Interfaces 1990, 20, 74-94.

19. Glover, F.; Laguna, M. Tabu Search; Kluwer Academic Publishers: Boston, MA, USA, 1997.

20. Ryan, J.; Bailey, T.; Moore, J. Reactive Tabu Search in Unmanned Aerial Reconnaissance Simulations. In Proceedings of the IEEE Winter Simulation Conference, Washington, DC, USA, 13-16 December 1998.

21. Wang, Z.; Liu, Q.; Tao, H.; Li, J. Multiple Task Planning Based on TS Algorithm for Multiple Heterogenous Unmanned Aerial Vehicles. In Proceedings of the IEEE Chinese Guidance, Navigation and Control Conference, Yantai, China, 8-10 August 2014.

22. Zhao, J.; Zhao, J. Study on Multi-UAV Task clustering and Task Planning in Cooperative Reconnaissance. In Proceedings of the International Conference on Intelligent Human-Machine Systems and Cybernetics, Hangzhou, China, 26-27 August 2014.

23. Dijkstra, E. A Note on Two Problems in Connexion with Graphs. Numer. Math. 1959, 1, $269-271$.

24. Meng, B.; Gao, X.; Wang, Y. Multi-mission Path Re-planning for Multiple Unmanned Aerial Vehicles Based on Unexpected Events. In Proceedings of the International Conference on Intelligent Human-Machine Systems and Cybernetics, Hangzhou, China, 26-27 August 2009; pp. 423-426.

25. Bertuccelli, L.; Choi, H.; Cho, P.; How, J. Real-time Multi-UAV Task Assignment in Dynamic and Uncertain Environments. In Proceedings of the AIAA Guidance, Navigation and Control Conference, Chicago, IL, USA, 10-13 August 2009.

26. Leary, S.; Deittert, M.; Bookless, J. Constrained UAV Mission Planning: A Comparison of Approaches. In Proceedings of the IEEE International Conference on Computer Vision, Barcelona, Spain, 6-13 November 2011; pp. 2002-2009.

27. Ragi, S.; Chong, E.K.P. Decentralized Guidance Control of UAVs with Explicit Optimization of Communications. J. Intell. Robot. Syst. 2014, 73, 811-822.

28. Cummings, M.; How, J.; Whitten, A.; Toupet, O. The Impact of Human-Automation Collaboration in Decentralized Multiple Unmanned Vehicle Control. Proc. IEEE 2012, 100, 660-671.

29. Kopeikin, A.; Ponda, S.; Johnson, L.; How, J. Multi-UAV Network Control Through Dynamic Task Allocation: Ensuring Data-Rate and Bit-Error-Rate Support. In Proceedings of the IEEE Globecom Workshop, Anaheim, CA, USA, 3-7 December 2012; pp. 1579-1584. 
30. Tong, H.; Wen, W.; Chang, H.; Yong, X. Path Planning of UAV Based on Voronoi Diagram and DPSO. In Proceedings of the International Workshop on Information and Electronics Engineering (IWIEE), Harbin, China, 10-11 March 2012; pp. 4198-4203.

31. Mattingley, J.; Wang, Y.; Boyd, S. Receding Horizon Control. Control Syst. IEEE 2012, 31, 52-65.

32. Kuwata, Y.; Richards, A.; Schouwenaars, T.; How, J. Decentralized Robust Receding Horizon Control for Multi-vehicle Guidance. In Proceedings of the American Control Conference, Minneapolis, MN, USA, 14-16 June 2006.

33. Kuwata, Y.; How, J. Robust Cooperative Decentralized Trajectory Optimization using Receding Horizon MILP. In Proceedings of the American Control Conference, New York, NY, USA, 11-13 July 2007.

34. Xiao, X.; Dong, Z.; Wu, J.; Duan, H. A Cooperative Approach to Multiple UAVs Searching for Moving Targets Based on a Hybrid of Virtual Force and Receding Horizon. In Proceedings of the IEEE 10th International Conference on Industrial Informatics, Beijing, China, 25-27 July 2012; pp. 1228-1233.

35. Peng, H.; Su, F.; Bu, Y.; Zhang, G.; Shen, L. Cooperative Area Search for Multiple UAVs based on RRT and Decentralized Receding Horizon Optimization. In Proceedings of the 7th Asian Control Conference, Hong Kong, China, 27-29 August 2009.

36. Schouwenaars, T.; How, J.; Feron, E. Decentralized Cooperative Trajectory Planning of Multiple Aircraft with Hard Safety Guarantees. In Proceedings of the AIAA Guidance, Navigation and Control Conference, Providence, RI, USA, 16-19 August 2004.

37. Miller, S.; Harris, Z.; Chong, E.K.P. A POMDP Framework for Coordinated Guidance of Autonomous UAVs for Multitarget Tracking. EURASIP J. Adv. Signal Process. 2009, 2009, 1-17.

38. Ragi, S.; Tan, C.; Chong, E.K.P. Feasibility Study of POMDP in Autonomous Amphibious Vehicle Guidance. In Proceedings of the IFAC Intelligent Autonomous Vehicles Symposium, Gold Coast, Australia, 26-28 June 2013.

39. Ragi, S.; Chong, E.K.P. UAV Path Planning in a Dynamic Environment via Partially Observable Markov Decision Process. IEEE Trans. Aerosp. Electron. Syst. 2013, 49, 2397-2412.

40. Kennedy, J.; Eberhart, R. Particle Swarm Optimization. In Proceedings of the IEEE International Conference on Neural Networks, Piscataway, NJ, USA, 27 November-1 December 1995; pp. 1942-1948.

41. Wang, G.; Li, Q.; Guo, L. Multiple UAVs Routes Planning Based on Particle Swarm Optimization Algorithm. In Proceedings of the 2nd International Symposium on Information Engineering and Electronic Commerce (IEEC), Ternopil, Ukraine, 23-25 July 2010.

42. Kenefic, R. Finding Good Dubins Tours for UAVs Using Particle Swarm Optimization. J. Aerosp. Comput. Inf. Commun. 2008, 5, 47-56.

43. Zhao, J.; Zhao, J. Target Distributing of Multi-UAVs Cooperative Attack and Defend Based on DPSO Algorithm. In Proceedings of the Sixth International Conference on Intelligent Human-Machine Systems and Cybernetics, Hangzhou, China, 26-27 August 2014; pp. 396-400.

44. Alejo, D.; Cobano, A.; Heredia, G.; Ollero, A. Collision-free Trajectory Planning Based on Maneuver Selection-Particle Swarm Optimization. In Proceedings of the International Conference on Unmanned Aircraft Systems (ICUAS), Denver, CO, USA, 9-12 June 2015.

45. Roberge, V.; Tarbouchi, M.; Labonte G. Comparison of Parallel Genetic Algorithm and Particle Swarm Optimization for Real-Time UAV Path Planning. IEEE Trans. Ind. Inform. 2013, 9, 132-141.

46. Sahingoz, O.K. Flyable Path Planning for a Multi-UAV System with Genetic Algorithms and Bezier Curves. In Proceedings of the International Conference on Unmanned Aircraft Systems (ICUAS), Atlanta, GA, USA, 28-31 May 2013; pp. 41-48.

47. Sahingoz, O.K. Generation of Bezier Curve-Based Flyable Trajectories for Multi-UAV Systems with Parallel Genetic Algorithm. J. Intell. Robot. Syst. Theory Appl. 2014, 74, 499-511.

48. Cekmez, U.; Ozsiginan, M.; Sahingoz, O.K. Adapting the GA Approach to Solve Traveling Salesman Problems on CUDA Architecture. In Proceedings of the IEEE International Symposium on Computational Intelligence and Informatics (CINTI 2013), Budapest, Hungary, 19-21 November 2013; pp. 423-428.

49. Cekmez, U.; Ozsiginan, M.; Sahingoz, O.K. A UAV Path Planning with Parallel ACO Algorithm on CUDA Platform. In Proceedings of the International Conference on Unmanned Aircraft Systems (ICUAS), Orlando, FL, USA, 27-30 May 2014; pp. 347-354.

50. Cheng, Z.; Sun, Y.; Liu, Y. Path Planning Based on Immune Genetic Algorithm for UAV. In Proceedings of the International Conference on Electric Information and Control Engineering (ICEICE), Wuhan, China, 15-17 April 2011; pp. 590-593. 
51. Price, I.; Lamont, G. GA Directed Self-Organized Search and Attack UAV Swarms. In Proceedings of the Winter Simulation Conference, Monterey, CA, USA, 3-6 December 2006; pp. 1307-1315.

52. Pehlivanoglu, Y. A New Vibrational Genetic Algorithm Enhanced with a Voronoi Diagram for Path Planning of Autonomous UAV. Aerosp. Sci. Technol. 2012, 16, 47-55.

53. Sonmez, A.; Kocyigit, E.; Kugu, E. Optimal Path Planning for UAVs Using Genetic Algorithm. In Proceedings of the International Conference on Unmanned Aircraft Systems (ICUAS), Denver, CO, USA, 9-12 June 2015.

54. Geng, L.; Zhang, Y.F.; Wang, J.J.; Fuh, J.Y.H.; Teo, S.H. Cooperative Task Planning for Multiple Autonomous UAVs with Graph Representation and Genetic Algorithm. In Proceedings of the 10th IEEE International Conference on Control and Automation (ICCA), Hangzhou, China, 12-14 June 2013.

55. Kirkpatrick, S.; Gelett, C.D.; Vecchi, M.P. Optimization by Simulated Annealing. Science 1983, 220, 621-630.

56. Cerny, V. A Thermodynamic Approach to the Traveling Salesman Problem: An Efficient Simulation. J. Optim. Theory Appl. 1985, 45, 41-51.

57. Turker, T.; Sahingoz, O.K.; Yilmaz, G. 2D Path Planning for UAVs in Radar Threatening Environment using Simulated Annealing Algorithm. In Proceedings of the International Conference on Unmanned Aircraft Systems (ICUAS), Denver, CO, USA, 9-12 June 2015.

58. Doringo, M. Optimization, Learning and Natural Algorithms. Ph.D. Thesis, Politecnico di Milano, Milan, Italy, 1992.

59. Fallahi, K.; Leung, H.; Chandana, S. An Integrted ACO-AHP Approach for Resource Management Optimization. In Proceedings of the IEEE International Conference on Systems, Man, and Cybernetics, San Antonio, TX, USA, 11-14 October 2009.

60. Duan, H.; Zhang, X.; Wu, J.; Ma, G. Max-Min Adaptive Ant Colony Optimization Approach to Multi-UAVs Coordinated Trajectory Replanning in Dynamic and Uncertain Envirionments. J. Bionic Eng. 2009, 6, 161-173.

61. Qu, Y.; Zhang, Y.; Zhang, Y. A UAV Solution of Regional Surveillance Based on Pheromones and Artificial Potential Field Theory. In Proceedings of the International Conference on Unmanned Aircraft Systems (ICUAS), Denver, CO, USA, 9-12 June 2015.

62. Zhang, Y.; Xiao, Y. A Patrolling Scheme in Wireless Sensor and Robot Networks. In Proceedings of the IEEE Conference on Computer Communications Workshops, Shanghai, China, 10-15 April 2011; pp. 513-518.

63. Zhang, Y.; Xiao, Y. Digital Pheromone Based Patrolling Algorithm in Wireless Sensor and Actuator Networks. In Proceedings of the IEEE Consumer Communications and Networking Conference, Las Vegas, NV, USA, 11-14 January 2013; pp. 496-501.

64. Shang, K.; Karungaru, S.; Feng, Z.; Ke, L.; Terada, K. A GA-ACO Hybrid Algorithm for the Multi-UAV Mission Planning Problem. In Proceedings of the Technologies (ISCIInternational Symposium on Communications and Information T), Incheon, Korea, 24-26 September 2014; pp. 243-248.

65. Krishnamoorthy, K.; Casbeer, D.; Pachter, M. Minimum Time UAV Pursuit of a Moving Ground Target using Partial Information. In Proceedings of the International Conference on Unmanned Aircraft Systems (ICUAS), Denver, CO, USA, 9-12 June 2015.

66. Krishnamoorthy, K.; Casbeer, D.; Chandler, P.; Pachter, M.; Darbha, S. UAV Search \& Capture of a Moving Ground Target under Delayed Information. In Proceedings of the IEEE Conference on Decision and Control, Mauai, Hawaii, USA, 10-13 December 2012.

67. Rasmussen, S.; Kingston, D. Development and Flight Test of an Area Monitoring System Using Unmanned Aerial Vehicles and Unattended Ground Sensors. In Proceedings of the International Conference on Unmanned Aircraft Systems (ICUAS), Denver, CO, USA, 9-12 June 2015.

68. Moon, S.; Yang, K.; Gan, S.; Shim, D. Decentralized Information-theoretic Task Assignment for Searching and Tracking of Moving Targets. In Proceedings of the International Conference on Unmanned Aircraft Systems (ICUAS), Denver, CO, USA, 9-12 June 2015.

69. Sun, A.; Liu, H. Cooperative UAV Search for Moving Targets Using a Modified Diffusion Uncertainty Model. In Proceedings of the AIAA Guidance, Navigation, and Control Conference, Chicago, IL, USA, 10-13 August 2009.

70. Frew, E.; Lawrence, D.; Morris, S. Coordinated Standoff Tracking of Moving Targets Using Lyapunov Guidance Vector Fields. J. Guid. Control Dyn. 2008, 31, 290-306. 
71. Summers, T.; Akella, M. Coordinated Standoff Tracking of Moving Targets: Control Laws and Information Architectures. In Proceedings of the AIAA Guidance, Navigation, and Control Conference and Exhibit, Honolulu, HI, USA, 18-21 August 2008.

72. Summers, T.; Akella, M.; Mears, M. Coordinated Standoff Tracking of Moving Targets: Control Lawas and Information Architectures. J. Guid. Control Dyn. 2009, 32, 56-69.

73. Geyer, C. Active Target Search from UAVs in Urban Environments. In Proceedings of the IEEE International Conference on Robotics and Automation, Pasadena, CA, USA, 19-23 May 2008.

74. Bertuccelli, L.; How, J. UAV Search for Dynamic Targets with Uncertain Motion Models. In Proceedings of the IEEE Conference on Decision \& Control, San Diego, CA, USA, 13-15 December 2006.

75. Ragi, S.; Chong, E.K.P. UAV Guidance Algorithms via Partially Observable Markov Decision Processes. In Handbook of Unmanned Aerial Vehicles; Valvanis, K., Vachtsevanos, G., Eds.; Springer: Dordrecht, The Netherlands, 2015; pp. 1775-1810.

76. Bertuccelli, L.F.; How, J.P. Robust UAV Search for Environments with Imprecise Probability Maps. In Proceedings of the IEEE Conference on Decision and Control, and the European Control Conference, Seville, Spain, 12-15 December 2005.

77. Song, S.; Rodriguez, A.; Teodorescu, M. Trajectory Planning for Autonomous Nonholonomic Vehicles for Optimal Monitoring of Spatial Phenomena. In Proceedings of the International Conference on Unmanned Aircraft Systems (ICUAS), Denver, CO, USA, 9-12 June 2015.

78. Lee, S.; Morrison, J. Decision Support Scheduling for Maritime Search and Rescue Planning with a system of UAVs and Fuel Service Stations. In Proceedings of the International Conference on Unmanned Aircraft Systems (ICUAS), Denver, CO, USA, 9-12 June 2015.

79. Zhang, C.; Pei, H. Oil Spills Boundary Tracking Using Universal Kriging and Model Predictive Control by UAV. In Proceedings of the 11th World Congress on Intelligent Control and Automation, Shenyang, China, 29 June-4 July 2014.

80. Hu, J.; Xie, L.; Lum, K.; Xu, J. Multiagent Information Fusion and Cooperative Control in Target Search. IEEE Trans. Control Syst. Technol. 2013, 21, 1223-1235.

81. Hirsch, M.; Schroeder, D. On the Decentralized Cooperative Control of Multiple Autonomous Vehicles. In Handbook of Unmanned Aerial Vehicles; Valvanis, K., Vachtsevanos, G., Eds.; Springer: Dordrecht, The Netherlands, 2015; pp. 1775-1810.

82. Hirsch, M.; Schroeder, D. Dynamic Decentralized Cooperative Control of Multiple Autonomous Vehicles with Multiple Tasks for Urban Operations. In Proceedings of the AIAA Guidance, Navigation, and Control Conference, Minneapolis, MN, USA, 13-16 August 2012.

83. Wu, P.; Campbell, D.; Merz, T. On-Board Multi-Objective Mission Planning for Unmanned Aerial Vehicles. In Proceedings of the IEEE Aerospace Conference, Piscataway, NJ, USA, 7-14 March 2009; pp. 1-10.

84. Ilaya, O. Multi Objective Decentralized Model Predictive Control for Cooperative Multi-UAV Systems. In Proceedings of the AIAA Guidance, Navigation and Control Conference, Hilton Head, SC, USA, 20-23 August 2007.

85. Ilaya, O.; Bil, C.; Evans, M. Distributed and Cooperative Decision Making for Multi-UAV Systems with Applicaiton to Collaborative Electronic Warfare. In Proceedings of the AIAA Aviation Technology, Integration and Operations Conference, Belfast, Northern Ireland, 18-20 September 2007.

86. Peng, X.; Xu, D.; Yan, W. Intelligent Flight for UAV via Integration of Dynamic MOEA, Bayesian Network and Fuzzy Logic. In Proceedings of the 50th IEEE Conference on Decision and Control and European Control Conference, Orlando, Fl, USA, 12-15 December 2011.

87. Chapman, A.; Mesbahi, M. UAV Swarms: Models and Effective Interfaces. In Handbook of Unmanned Aerial Vehicles; Valvanis, K., Vachtsevanos, G., Eds.; Springer: Dordrecht, The Netherlands, 2015; pp. 1775-1810.

88. Barca, J.; Sekercioglu, Y. Swarm robotics reviewed. Robotica 2013, 31, 345-359.

89. Alighanbari, M.; How, J. An Unbiased Kalman Consensus Algorithm. In Proceedings of the American Control Conference, Minneapolis, MN, USA, 14-16 June 2006.

90. Brunet, L.; Choi, H.; How, J. Consensus-Based Auction Approaches for Decentralized Task Assignment. In Proceedings of the AIAA Guidance Navigation and Control Conference, Honolulu, HI, USA, 18-21 August 2008.

91. Choi, H.; Brunet, L.; How, J. Consensus-Based Decentralized Auctions for Robust Task Allocation. IEEE Trans. Robot. 2009, 25, 912-926. 
92. Choi, H.; Whitten, A.; How, J. Decentralized Task Allocation for Heterogeneous Teams with Cooperation Constraints. In Proceedings of the 2010 American Control Conference, Baltimore, MD, USA, 30 June-2 July 2010.

93. Ren, W.; Beard, R.; Atkins, E. A Survey of Consensus Problems in Multi-agent Coordination. In Proceedings of the 2005 American Control Conference, Portland, OR, USA, 8-10 June 2005.

94. Ren, W.; Beard, R. Distributed Consensus in Multi-vehicle Cooperative Control; Springer-Verlag: London, UK, 2008.

95. Ren, W.; Beard, R. Consensus Seeking in Multiagent Systems under Dynamically Changing Interaction Topologies. IEEE Trans. Autom. Control 2005, 50, 655-661.

96. Sujit, P.; Kingston, D.; Beard, R. Cooperative Forest Fire Monitoring Using Multiple UAVs. In Proceedings of the 46th IEEE Conference on Decision and Control, New Orleans, LA, USA, 12-14 December 2007.

97. Kingston, D.; Beard, R. Decentralized Perimeter Surveillance Using a Team of UAVs. IEEE Trans. Robot. 2008, 24, 1394-1404.

98. Ou, W.; Zou, F.; Xu, X.; Gao, Z. Targets Assignment for Cooperative Multi-UAVs Based on Chaos Optimization Algorithm. In Proceedings of the 9th International Conference for Young Computer Scientists, Hunan, China, 18-21 November 2008.

99. Zhang, B.; Mao, Z.; Liu, W.; Liu, J.; Zheng, Z. Cooperative and Geometric Learning for Path Planning of UAVs. In Proceedings of the 2013 International Conference on Unmanned Aircraft Systems (ICUAS), Atlanta, GA, USA, 18-21 May 2013.

100. Clark, M.; Koutsoukos, X.; Kumar, R.; Lee, I.; Pappas, G.; Pike, L.; Porter, J.; Sokolsky, O. A Study on Run Time Assurance for Complex Cyber Physical Systems; Air Force Research Lab: Dayton, OH, USA, 2013.

101. Zhang, X.; Clark, M.; Rattan, K.; Muse, J. Controller Verification in Adaptive Learning Systems Towards Trusted Autonomy. In Proceedings of the ACM/IEEE Sixth International Conference on Cyber-Physical Systems, Seattle, WA, USA, 14-16 April 2015.

102. Schierman, J.; DeVore, M.; Cooper, J.; Richards, N.; Gandhi, N.; Horneman, K.; Smolka, S.; Stoller, S.; Clark, M. Run Time Assurance for Complex Autonomy. In Proceedings of the Safe and Secure Systems and Software Symposium, Dayton, OH, USA, 9-11 June 2015.

103. Skoog, M. Expandable Variable-Autonomy Architecture. NASA Fact Sheet, 2015. Available online: http:/ / www.nasa.gov/centers/armstrong/news/FactSheets/index.html (accessed on 19 August 2015).

104. Ho, N.; Koltai, K.; Masequesmay, G.; Cals, S.; Sadler, G.; Lyons, J.; Cancanindin, A.; Johnson, W.; Skoog, M. An Ethnographic-Based Model for Trust Development in Auto-GCAS. In Proceedings of the American Psychological Associate Annual Convention, Toronto, ON, Canada, 6-9 August 2015.

105. Niedober, D.; Ho, N.; Koltai, K.; Masequesmay, G.; Skoog, M.; Cacanindin, A.; Johnson, W.; Lyons, J. Influence of Cultural, Organizational, and Automation Capability Factors on Human-Automation Trust: A Case Study of Auto-GCAS Engineers. In Proceedings of the International Conference on Human Computer Interaction, Crete, Greece, 22-27 June 2014.

106. Koltai, K.; Ho, N.; Masequesmay, G.; Niedober, D.; Skoog, M.; Cacanindin, A.; Johnson, W.; Lyons, J. Influence of Cultural, Organizational, and Automation Capability on Human Automation Trust: A Case Study of Auto-GCAS Experimental Test Pilots. In Proceedings of the International Conference on Human Computer Interaction in Aerospace, Santa Clara, CA, USA, 30 July-1 August 2014.

107. Koltai, K.; Ho, N.; Masequesmay, G.; Niedober, D.; Skoog, M.; Cacanindin, A.; Johnson, W.; Lyons, J. An Extended Case Study Methodology for Investigating Influence of Cultural, Organizational, and Automation Factors on Human-Automation Trust. In Proceedings of the ACM-CHI 2014 Conference, Toronto, ON, Canada, 26 April-1 May 2014.

108. Bice, G.; Skoog, M.; Howard, J. Aircraft Ground Collision Avoidance and Autorecovery System Device. U.S. Patent 4,924,401, 8 May 1990.

109. Skoog, M.; Prosser, K. Advanced Fighter Technology Integration/F-16 Automatic Ground Collision Avoidance System Evaluation; AFFTC-TR-99-28; Wright-Patterson Air Force Base: Dayton, OH, USA, 2000.

110. Moore, D.; Schlappi, K. USAF F-16 Block 40/50 Test and Evaluation for the Automatic Ground Collision Avoidance System (Auto GCAS) and Pilot Activated Recovery System (PARS); 412TW-TR-13-04; Wright-Patterson Air Force Base: Dayton, OH, USA, 2013.

111. Sorokowski, P. Automatic Ground Collision Avoidance System Fighter Risk Reduction Project; AFFTC-TIM-10-05; Wright-Patterson Air Force Base: Dayton, OH, USA, 2010. 
112. Swihart, D.E.; Barfield, A.F.; Griffin, E.M.; Lehmann, R.C.; Whitcomb, S.C.; Flynn, B.; Skoog, M.A.; Processor, K.E. Automatic Ground Collision Avoidance System design, integration, \& flight test. Aerosp. Electron. Syst. Mag. 2011, 26, 4-11.

113. Norris, G. Ground Collision Avoidance System "Saves" First F-16 in Syria. 5 February 2015. Available online: http:/ /aviationweek.com/defense/ground-collision-avoidance-system-saves-first-f-16-syria (accessed on 11 October 2015).

114. Sorokowski, P.; Skoog, M.; Burrows, S.; Thomas, S. Small UAV Automatic Ground Collision Avoidance System Design Considerations and Flight Test Results; NASA/TM-2015-218732; NASA: Washington, DC, USA, 2015.

115. Scherer, S.; Singh, S.; Chamberlain, L.; Elgersma, M. Flying Fast and Low Among Obstacles: Methodology and Experiments. Int. J. Robot. Res. 2008, 27, 549-574.

116. Kobilarov, M. Cross-entropy Motion Planning. Int. J. Robot. Res. 2012, 31, 855-871.

117. Hrabar, S. Reactive Obstacle Avoidance for Rotorcraft UAVs. In Proceedings of the Intelligent Robots and Systems Conference, San Francisco, CA, USA, 25-30 September 2011.

118. Wood, D. Collision Avoidance System and Method Utilizing Variable Surveillance Envelope. U.S. Patent 6,804,607, 12 October 2004.

119. Noth, K. Modeling and Simulation of a Ground Based Sense and Avoid Architecture for Unmanned Aircraft System Operations. In Proceedings of the Integrated Communications, Navigation and Surveillance Conference, Herndon, VA, USA, 11-13 May 2011.

120. Ottman, M. Army Ground Based Sense and Avoid (GBSAA): Enables Unmanned Aircraft Flight in the National Airspace. Available online: http://www.army.mil/article/80681/Army_Ground_Based_Sense_and_Avoid_GBSAA (accessed on 11 October 2015).

121. Campbell, P. Challenges to using Ground Based Sense and Avoid (GBSAA) for UAS Operations. In Proceedings of the Digital Avionic Systems Conference, Williamsburg, VA, USA, 14-18 October 2012.

122. Federal Aviation Administration. Automatic Dependent Surveillance-Broadcast (ADS-B). Available online: http://www.faa.gov/nextgen/programs/adsb/ (accessed on 11 October 2015).

123. Lin, Y.; Saripalli, S. Sense and Avoid for Unmanned Aerial Vehicles Using ADS-B. In Proceedings of the International Conference on Unmanned Aircraft Systems (ICUAS), Denver, CO, USA, 9-12 June 2015.

124. Liu, W.; Hwang, I. Probabilistic Aircraft Midair Conflict Resolution Using Stochastic Optimal Control. IEEE Trans. Intell. Transp. Syst. 2014, 15, 37-46.

125. Richards, A.; How, J. Aircraft Trajectory Planning With Collision Avoidance Using Mixed Integer Linear Programming. In Proceedings of the American Control Conference, Anchorage, AK, USA, 8-10 May 2002.

126. Patel, R.; Goulart, P.; Serghides, V. Real-Time Trajectory Generation for Aircraft Avoidance Maneuvers. In Proceedings of the AIAA Guidance, Navigation, and Control Conference, Chicago, IL, USA, 10-13 August 2009.

127. Lin, Y.; Saripalli, S. Collision Avoidance for UAVs Using Reachable Sets. In Proceedings of the International Conference on Unmanned Aircraft Systems (ICUAS), Denver, CO, USA, 9-12 June 2015.

128. Lin, Y.; Saripalli, S. Path Planning Using 3D Dubins Curve for Unmanned Aerial Vehicles. In Proceedings of the International Conference on Unmanned Aircraft Systems (ICUAS), Orlando, CA, USA, 27-30 May 2014.

129. Shim, D.; Sastry, S. An Evasive Maneuvering Algorithm for UAVs in See-and-Avoid Situations. In Proceedings of the American Control Conference, New York City, NY, USA, 11-13 July 2007.

130. Bareiss, D.; van den Berg, J. Reciprocal Collision Avoidance for Robots with Linear Dynamics using LQR-Obstacles. In Proceedings of the IEEE International Conference on Robotics and Automation, Karlsruhe, Germany, 6-10 May 2013.

131. Wolf, T.; Kochenderfer, M. Aircraft Collision Avoidance Using Monte Carlo Real-Time Belief Space Search. J. Intell. Robot. Syst. 2011, 64, 277-298.

132. Temizer, S.; Kochenderfer, M.; Kaelbling, L.; Lozano-Perez, T.; Kuchar, J. Collision Avoidance for Unmanned Aircraft using Markov Decsion Processes. In Proceedings of the AIAA Guidance, Navigation, and Control Conference, Toronto, ON, Canada, 2-5 August 2010.

133. Bai, H.; Hsu, D.; Kochenderfer, M.; Lee, W. Unmanned Aircraft Collision Avoidance Using Continuous-State POMDPs. In Robotics: Science and Systems; MIT Press: Cambridge, MA, USA, 2012; pp. 1-8.

134. Jackson, J.; Boskovic, J.; Diel, D. Sensor Fusion for Sense and Avoid for Small UAS without ADS-B. In Proceedings of the International Conference on Unmanned Aircraft Systems (ICUAS), Denver, CO, USA, 9-12 June 2015. 
135. Williamson, T.; Spencer, N. Development and Operation of the Traffic Alert and Collision Avoidance System (TCAS). Proc. IEEE 1989, 77, 1735-1744.

136. Wilson, M. The Use of Low-Cost Mobile Radar Systems for Small UAS Sense and Avoid. In Sense and Avoid in UAS Research and Applications; Angelov, P., Ed.; John Wiley and Sons: West Sussex, UK, 2012; pp. 295-336.

137. Griffith, J.D.; Kochenderfer, M.J.; Kuchar, J.K. Electro-optical system analysis for sense and avoid. In Proceedings of the AIAA Guidance, Navigation and Control Conference, Honolulu, HI, USA, 18-21 August 2008.

138. Ramasamy, S.; Gardi, A.; Liu, J.; Sabatini, R. A Laser Obstacle Detection and Avoidance System for Manned and Unmanned Aircraft Applicatins. In Proceedings of the International Conference on Unmanned Aircraft Systems (ICUAS), Denver, CO, USA, 9-12 June 2015.

139. Angelov, P. Sense and Avoid in UAS Research and Applications; Angelov, P., Ed.; John Wiley and Sons: West Sussex, UK, 2012; pp. 295-336.

140. Contarino, M. All Weather Sense and Avoid System for UASs Report. Task 3.1 - Review E Analysis of Available System Technology Options and Justification for System Selection. Technical Report; U.S. Office of Naval Research: Arlington, VA, USA, 2009.

141. Stevens, M.; Coloe, B.; Atkins, E. Platform-Independent Geofencing for Low Altitude UAS Operations. In Proceedings of the 15th Aviation Technology Integration, and Operations Conference, Dallas, TX, USA, 22-26 June 2015.

142. Arduino Autopilot Geo-Fencing. Available online: http://plane.ardupilot.com/wiki/geofencing/ (accessed on 13 September 2015).

143. Hayhurst, K.; Maddalon, J.; Neogi, N.; Verstynen, H. A Case Study for Assured Containment. In Proceedings of the International Conference on Unmanned Aircraft Systems (ICUAS), Denver, CO, USA, 9-12 June 2015.

144. Range Commanders Council. Flight Safety System (FSS) for Unmanned Aerial Vehicle (UAV) Operation; Special Report; U.S. Army: Washington, DC, USA, 2008.

145. Scheidt, D.; D'Amico, W.; Lutz, R. Safe Testing of Autonomy in Complex, Interactive Environments (TACE). ITEA J. 2014, 35, 323-331.

146. Stansbury, R.; Tanis, W.; Wilson, T. A Technology Survey of Emergency Recovery and Flight Termination Systems for UAS. In Proceedings of the AIAA Infotech Aerospace Conference, Seattle, WA, USA, 6-9 April 2009.

(C) 2016 by the authors; licensee MDPI, Basel, Switzerland. This article is an open access article distributed under the terms and conditions of the Creative Commons by Attribution (CC-BY) license (http:/ / creativecommons.org/licenses/by/4.0/). 